\title{
REVIEW ARTICLE Identifying adverse childhood experiences in pediatrics to prevent chronic health conditions
}

\author{
Andrew J. Barnes ${ }^{1}$, Bruno J. Anthony ${ }^{2}$, Canan Karatekin ${ }^{3}$, Katherine A. Lingras ${ }^{4}$, Rebeccah Mercado ${ }^{5}$ and Lindsay Acheson Thompson ${ }^{5}$
}

\begin{abstract}
Despite evidence that over $40 \%$ of youth in the United States have one or more adverse childhood experiences (ACEs), and that ACEs have cumulative, pernicious effects on lifelong health, few primary care clinicians routinely ask about ACEs. Lack of standardized and accurate clinical assessments for ACEs, combined with no point-of-care biomarkers of the "toxic stress" caused by ACEs, hampers prevention of the health consequences of ACEs. Thus, there is no consensus regarding how to identify, screen, and track ACEs, and whether early identification of toxic stress can prevent disease. In this review, we aim to clarify why, for whom, when, and how to identify ACEs in pediatric clinical care. To do so, we examine the evidence for such identification; describe the efficacy and accuracy of potential screening instruments; discuss current trends in, and potential barriers to, the identification of ACEs and the prevention of downstream effects; and recommend next steps for research, practice, and policy.
\end{abstract}

Pediatric Research (2020) 87:362-370; https://doi.org/10.1038/s41390-019-0613-3

\section{BACKGROUND}

Adverse childhood experiences (ACEs) are potentially traumatic events that cause overwhelming stress and have lasting negative effects on physical and mental health. ${ }^{1-4}$ Such experiences include abuse and maltreatment (physical, sexual, emotional/verbal); neglect (physical and emotional/psychological); and household dysfunction (parent mental illness; domestic violence; divorce or separation; incarceration; and alcohol or drug abuse). Other overwhelmingly stressful experiences that were not included in the original ACEs scale, but should be considered possible ACEs due to evidence for their effects on child development and health, include severe economic hardship, hunger, disabilities, medical trauma, war, disaster, homelessness, bullying victimization, and discrimination. ${ }^{5-13}$

This overwhelming stress is deemed "toxic" due to its associations with modified gene expression, increased allostatic load, and problems with cognitive and social-emotional development. ${ }^{14-16}$ Research dating back to the mid-20th century repeatedly implicates the cumulative effects of ACEs and the resultant effects of toxic stress on health outcomes across the lifespan. ${ }^{17}$ These effects include higher risk of mental illness, substance abuse, and suicide; diabetes; heart disease; and cancer in adulthood. ${ }^{18-21}$ Children who have experienced at least one ACE are at increased risk for chronic physical health problems during childhood adolescence (e.g., obesity, learning and developmental delays, and mental, emotional, and behavioral problems. ${ }^{2-27}$

Pediatric clinicians are increasingly called upon to identify children whose development and health may be at risk due to ACEs. While families may be willing to discuss a child's exposure to ACEs with a trusted health care professional, ${ }^{28}$ there is not a clear consensus regarding how to identify, screen, and track these experiences and whether early identification of toxic stress improves outcomes. In this review, we aim to clarify why, for whom, when, and how to identify ACEs in pediatric primary care. To do so, we will examine the evidence in favor of such identification; describe the efficacy and accuracy of potential screening instruments; discuss current trends and potential issues in the identification of toxic stress; and recommend next steps for research and practice.

\section{RATIONALE FOR IDENTIFICATION OF ACES IN PEDIATRIC HEALTH CARE}

Universal screening for ACEs in pediatric health care has been proposed as a way to systematically detect and inform interventions to address a variety health, developmental, and behavioral problems. For instance, the American Academy of Pediatrics (AAP) has issued policy statements and guidance on screening and surveillance in the medical home that recommends that clinicians identify risk and protective factors for developmental and mental disorders for all children and families in their care. ${ }^{29}$ However, there remain a number of challenges to fully implementing this AAP policy, including its lack of specificity and relatively shallow evidence base, likely contributing to the only modest shift in practice to proactively identify and treat trauma exposure. ${ }^{30,31}$ Clinicians do, however, routinely learn about life circumstances that effect children's health during conversations that take place within high-quality patient-clinician relationships. Given that these relationships and therapeutic conversations are themselves the foundation for trauma-informed care, "surveillance" for ACEs could be a natural part of pediatric health care given appropriate support within clinical systems. ${ }^{32}$ Similarly, AAP's 2012 policy and accompanying technical report on ACEs discusses the need for systematic screening of children and their families who are at risk

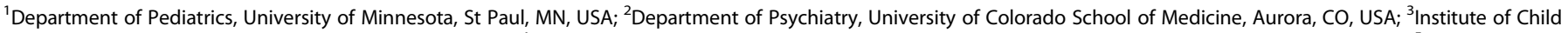

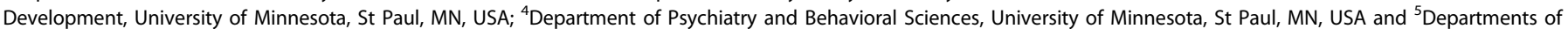
Pediatrics, Health Outcomes and Policy, University of Florida, Gainesville, FL, USA

Correspondence: Andrew J. Barnes (drbarnes@umn.edu)

Received: 22 April 2019 Revised: 16 August 2019 Accepted: 25 September 2019

Published online: 17 October 2019 


\begin{tabular}{|l|c|c|c|c|}
\hline $\begin{array}{l}\text { Example } \\
\text { screenings }\end{array}$ & $\begin{array}{c}\text { Single } \\
\text { outcome } \\
\text { measure(s) }\end{array}$ & $\begin{array}{c}\text { Clear cutoff } \\
\text { value(s) }\end{array}$ & $\begin{array}{c}\text { Endorsed by } \\
\text { bright futures }\end{array}$ & $\begin{array}{c}\text { Clinical } \\
\text { guidelines for } \\
\text { intervention/ } \\
\text { treatment }\end{array}$ \\
\hline $\begin{array}{l}\text { Anemia } \\
\text { screening (lab } \\
\text { test) }\end{array}$ & $\checkmark$ & $\checkmark$ & $\checkmark$ & $\checkmark$ \\
\hline $\begin{array}{l}\text { Hypertension } \\
\text { (vital sign) }\end{array}$ & $\checkmark$ & $\checkmark$ & $\checkmark$ & $\checkmark$ \\
\hline $\begin{array}{l}\text { Developmental } \\
\text { delay } \\
\text { (standardized } \\
\text { parent-report) }\end{array}$ & $\mathrm{X}$ & $\begin{array}{c}\text { (Screener } \\
\text { dependent) }\end{array}$ & $\checkmark$ & $\checkmark$ \\
\hline $\begin{array}{l}\text { Adverse } \\
\text { childhood } \\
\text { experiences } \\
\text { (methods vary) }\end{array}$ & $\mathrm{X}$ & $\mathrm{X}$ & $\checkmark$ & $\mathrm{X}$ \\
\hline
\end{tabular}

Fig. 1 Common screening measures in pediatrics compared to ACE identification

of toxic stress. ${ }^{23}$ In fact, some ACEs themselves are preventable and/or can be ameliorated by early intervention efforts. For instance, programs such as the nurse-family partnership, head start and/or preschool programs, and parenting programs (e.g., circles of security, attachment-biobehavioral catch-up, positive parenting program aka triple $P$ ) show long-term impacts for ameliorating risk among children and families who have experienced risk and ACEs. For children who have experienced ACEs, negative health outcomes may be mitigated in the presence of adequate protective factors, whether internal (such as selfregulation or resilience) or external (such as a consistent, nurturing adult caregiver, or strong community connectedness). For example, having a caring and supportive relationship with a teacher and/or adult caregiver helps a child develop problemsolving skills, critical thinking, autonomy, critical consciousness, and a sense of purpose. Such relationships provide a foundation for healthy development under stressful conditions. ${ }^{33}$ Protective factors that buffer against the toxic stress of ACEs thus improve lifelong resilience. ${ }^{34,35}$ Indeed, the toxic stress of ACEs-such as hypothalamic-pituitary axis perturbations-can be reversed with appropriate early intervention, such as enriched foster care that includes intensive parenting support. ${ }^{36,37}$

In pediatric clinical care, ACEs are at least as prevalent as other conditions for which standardized screening is already recommended (e.g., anemia, hypertension, cholesterol, developmental delay, and emotional-behavioral disorders)-for example, $67 \%$ of youth in an urban clinic had at least $1 \mathrm{ACE}$, and $12 \%$ had 4 or more. $^{38}$ Nationally, findings from the 2016 National Survey of Children's Health estimate that nearly half $(46 \%)$ of US children 0-17 years old have experienced at least one ACE with almost one-third (29.9\%) of US children 12-17 years old having experienced two or more ACEs. ${ }^{39}$ Identifying ACEs in clinical settings could thus be of high yield, yet it remains difficult to implement systemically.

Despite the evidence in favor of identifying ACEs, one-third of AAP members never inquire about ACEs; of those who do, many are not aware of appropriate screening tools or do not use them. ${ }^{30}$ Most pediatricians who inquire about ACEs are assessing for maternal depression ${ }^{30}$ (for which validated screeners exist ${ }^{40}$ ), whereas other common ACEs such as parental alcohol/drug use, domestic violence, and incarceration are rarely discussed. Clinicians may hesitate to surveil or screen for ACEs for reasons similar to other under-identified conditions such as emotional-behavioral disorders-for example, lack of training, lack of time, lack of referral resources, and limited awareness of how to address concerns that are raised in the process of screening (i.e., lack of confidence in discussing trauma and resilience). ${ }^{41-44}$ Furthermore, pediatric ACE identification through standardized screening may have unique challenges compared to other childhood screenings (Fig. 1)-for example, the medical model focuses on single-outcome "cutpoints" to guide the provision of specific biomedical interventions, whereas ACE screening identifies social and psychological determinants of health that may be more subjective (lacking evidence of cut-points for "toxicity") and interventions that current health care systems may not yet adequately deliver. ${ }^{45}$ In childhood lead screening, for example, quantifiable abnormalities are well established and associated with specific interventions and clinical algorithms. Even for parent-reported screenings, such as for child development and behavior, there are clear best practices, "pass/ fail" standards, and interventions. However, these standards and guidelines are not yet available for ACEs, which might defy screening as currently practiced in the medical model due to their psychosocial complexity. For these reasons, ACE screeners may not fully meet the World Health Organization principles for screening. $^{46}$

Methodological challenges to standardized ACE screening in pediatric health care include differing opinions on which adversities to screen for; when to screen; excessive burden of existing screenings; time constraints within the clinic; and what type of intervention or treatment to provide and how and when to provide it. ${ }^{4-50}$ Further, because existing ACE screeners provide limited information about the context of any given adverse event (e.g., timing, duration, involvement of protective caregivers), it is difficult to draw accurate conclusions based on a screen in which experiences are endorsed or denied without additional details about context. Furthermore, the perception of whether an ACE is stressful or worthy of intervention differs between individuals and cultures, so patients and families may find such screening intrusive or irrelevant. Additionally, clinics may not have a system in place for referral of mental health/social concerns that are reported in the context of ACE screening, thus placing providers in a difficult position of not knowing what to do with sensitive information revealed by families.

In summary, despite their prevalence, health burden, and effective prevention and treatment strategies, to date there is scant evidence to guide the collection of information about ACEs in pediatric health care. Prevention of ACEs and early interventions for resultant toxic stress thus hinge on identifying ACEs using methods that are feasible, efficient, accurate, actionable, and trackable. 


\section{CURRENT METHODS FOR IDENTIFYING ACES}

Over the past decade, pediatric researchers increasingly highlighted the importance of identifying child maltreatment in order to better support positive child development. ${ }^{51,52}$ Through the Bright Futures guidelines for well-child preventive care, the AAP recommends developmental surveillance and psychosocial/behavioral assessments at all well-child checks from newborn to adulthood. While not specific to ACEs, these surveillance touch points are unstructured and potentially vast, covering topics from depression to child abuse. For higher-risk groups such as children in foster care, the AAP has suggested ways in which clinicians can incorporate surveillance for ACEs into routine primary care, including asking questions such as, "Do you know of any really scary or upsetting things that happened to you/your child either before or after he/she came to live with you?" and "Since the last time I saw you/your child, has anything really scary or upsetting happened to you/your child or anyone in the family?" These and other open-ended verbal surveillance questions remain unstudied in terms of their clinical or public health utility, but are likely to complement any efforts towards standardized ACE screening, especially given the current limitations of putative ACE screeners.

Several self-report measures are available to screen youth for trauma (abuse/violence and/or neglect)-including the Childhood Trauma Questionnaire; the Structured Trauma-Related Experiences and Symptoms Screener; the Trauma Symptom Checklist for Children; and the Juvenile Victimization Questionnaire (JVQ) - 2nd Revision. ${ }^{53-56}$ While some of these screens such as the JVQ ask about a broad array of experiences and have been used in large epidemiological studies, others are specific to trauma or posttraumatic stress disorder, most have been normed only in small samples of referred youth, and some are not validated for parent report or for children under age 8 years, although recent work with adaptations of some measures such as the Traumatic Events Screening Inventory for Children (TESI-C) show promise. ${ }^{57}$ Some measures, such as the adapted TESI-C or the Survey of Well-Being in Young Children (SWYC), ${ }^{58}$ also screen for emotional-behavioral symptoms; others, such as the Center for Youth Wellness Adverse Childhood Experiences Questionnaires (CYW-ACEQ) ${ }^{59}$ incorporate symptoms into their guidance to the clinician regarding interpretation of ACE "scores." Parent-report measures that include other ACEs and may be suitable for screening in pediatrics are summarized in Table 1. Some of these (and others) have recently been reviewed in detail by $\mathrm{Oh}$ et al. ${ }^{60}$

The sensitivity of the original CDC ACE questions may be improved in pediatric settings by adding questions about economic hardship and food insecurity ${ }^{61}$ however, such an adaptation of the CDC ACE questions has not yet been studied as a parent-report screener in pediatric clinical settings. The Safe Environments for Every Kid Parent Questionnaire-Revised (SEEK $P Q-R)$ and Well-child care, Evaluation, Community resources, Advocacy, Referral, Education (WE-CARE (WE-CARE) screeners seem most promising for use in pediatric clinical care because they have demonstrated positive outcomes in RCTs. Parents who were screened with the SEEK PQ by primary care clinicians trained to make effective referrals showed reductions in child maltreatment, ${ }^{62}$ and those screened with WE-CARE showed higher rates of contact with community resources, referrals, and topics discussed with the clinician. ${ }^{63,64}$ From a psychometric standpoint, there is no current consensus as to what constitutes gold-standard criteria by which to measure the sensitivity and specificity of these or other candidate ACE screeners, and even trauma-specific screeners lack established norms. ${ }^{65,66}$ However, responding to the possibility of false-negative screens, the creators of the SEEK PQ-R appropriately point out on their website that "It's possible that by asking the question(s), [the clinician has] shown [his or her] interest, and sown a seed. [Parents] may disclose in the future. Parents who choose not to disclose are probably not amenable to intervention at this time. ${ }^{\prime 67}$ Finally, as noted above, practice barriers to implementing ACE screening are substantial, especially given that broader recommended developmental, behavioral, and mental health screenings are not yet universally implemented.

\section{EMERGING TRENDS AND ISSUES}

As mentioned above, concepts of risk and resilience are not new, and evidence has mounted over decades speaking to the multilevel effects of ACEs and other adverse circumstances during childhood. Concurrently, evidence has accumulated that informs prevention of childhood toxic stress, along with treatment for those who have experienced it. Furthermore, the past decade has brought rapid advances to pediatrics: integrated behavioral care; comprehensive and robust electronic health records (EHRs); and inter-professional training and cross-sector collaboration. Taken as a whole, these advances allow clinicians to identify and track risk and/or protective factors during childhood better than could have been done in the past. Nevertheless, the science of ACEs and technological advances alone provide inadequate justification for individual-level ACE screening. Many caveats remain, such as a lack of understanding about the role of the patient-clinician relationship in the assessment of risk and protective factors; potential unintended consequences of individual screening (e.g., loss of patient trust from "false positives" and/or insensitively delivered screening); whether or how to account for the context of ACEs such as timing and severity; the role of cultural differences; and how screening for ACEs may inadvertently overlook or even perpetuate factors that may underly ACEs such as racism and historical trauma. These factors are outside the scope of this paper, but are reviewed concisely elsewhere. ${ }^{68}$ Thus, routine and accurate identification of ACEs and related risk factors in pediatrics will require innovative and readily implementable solutions, as well as new research; we detail several emerging areas that deserve further attention.

One way to educate about and foster implementation of ACE screening, for example, would be a Maintenance of Certification or Quality Improvement project, which could be accomplished through AAP EQIPP (American Academy of Pediatrics Education in Quality Improvement for Pediatric Practice) ${ }^{69}$ or American Board of Pediatrics Performance Improvement Modules. ${ }^{70}$ These activities allow clinicians to put knowledge into action, encourage a team approach, and promote incremental practice change. Other trauma-informed projects aimed a practice improvement, such as the Pediatric Integrated Care Collaborative, ${ }^{71}$ could also produce new knowledge that could inform refinement of existing screening tools or creation of new methods. Provider/clinician training in ACE identification may also vary drastically depending on which role is tasked with screening-for example, nurse, medical assistant, physician-and whether screening is a review of a previously completed questionnaire (e.g., online) or an in-person conversation. In the case of an in-person conversation, the importance of long-term therapeutic relationships and traumainformed care cannot be understated-the effects of this kind of care on children deserves ongoing study. ${ }^{72}$

To aid in clinical decision-making and long-term tracking of ACEs, it is important to consider integration of ACE screening and/ or surveillance into the EHR. One promising idea is to use the EHR for more automated ACE identification, using technological solutions such as natural language processing of free-text fields or automatically flagging diagnostic codes indicative of ACEshowever, such approaches may lead to false positives due to technological limitations, while also failing to adequately reflect the true prevalence of ACEs given that clinicians may underidentify ACEs such as child maltreatment in EHRs. ${ }^{73}$ Potentially, EHRs would provide an alert to the clinician when some form of ACE screen is positive, with suggested language for helping 


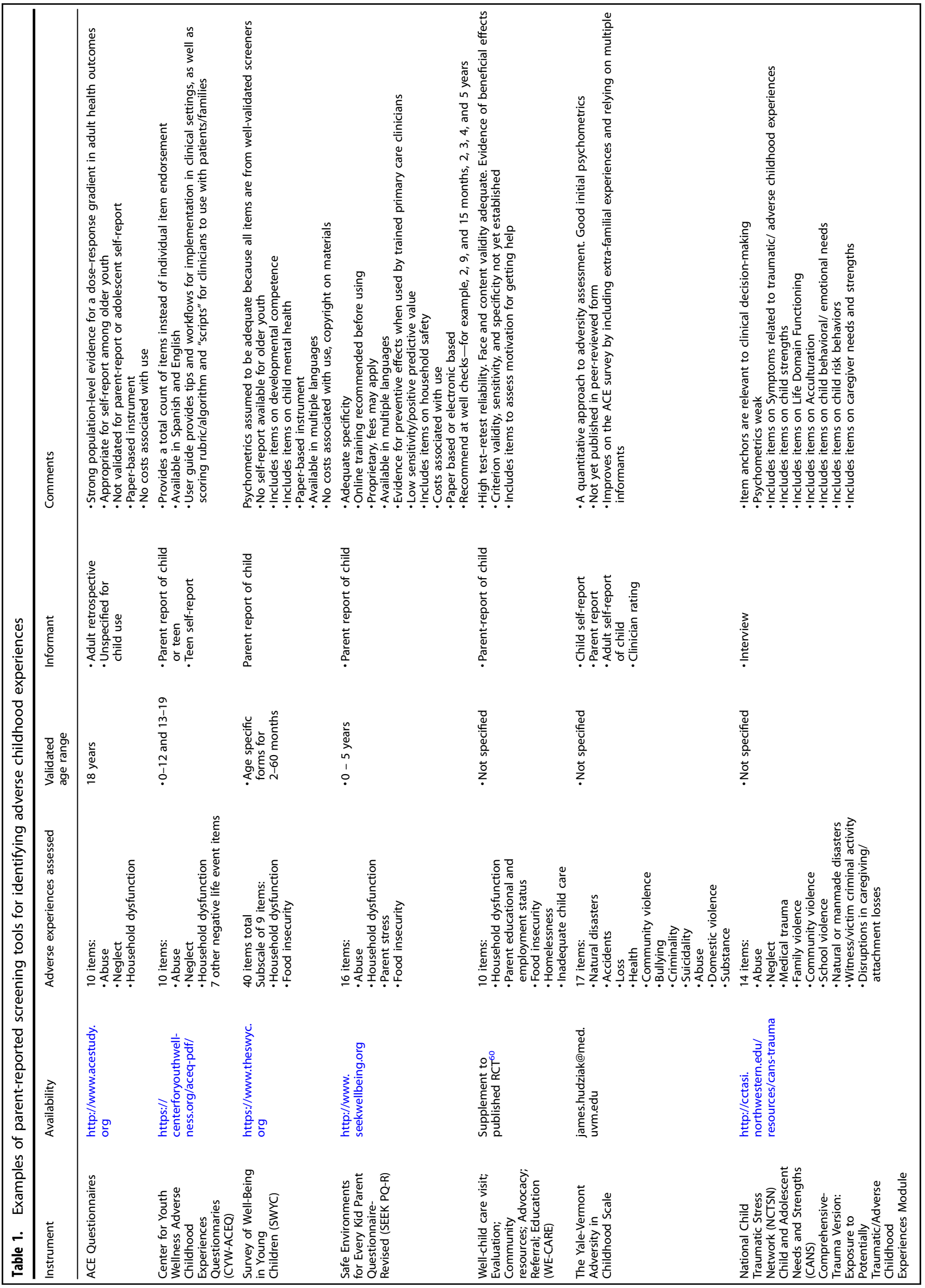


families understand how life experiences shape brain development and health, asking the patient/family if help is wanted, and providing referrals to the appropriate resource or service. Similarly, clinicians might screen for one or more potential ACEs (or proxies for ACEs) that do not require mandated reporting, as do maltreatment or neglect-for example, hunger/food insecurity, economic hardship or financial stress, housing instability, or family dysfunction-and for those who screen positive, perform expanded screening themselves (e.g., for other ACEs), or refer to social/behavioral clinical team members integrated within their systems of care.

Novel methods of identifying ACEs, such as parent ACEs, ${ }^{74}$ or even identifying toxic stress itself via altered salivary biomarkers, ${ }^{75}$ may seem to hold promise based on their correlation with child and adolescent ACEs across numerous studies. Other measures, including various biological moderators of individual differences in sensitivity to experiences, ${ }^{76}$ may someday be useful (e.g., to know which prevention and intervention strategies work best for individual children). However, these methods remain squarely in the domain of research investigation, and for now remain impractical, infeasible, and uninterpretable for individual patients in clinical practice.

Screening for protective factors that help foster resilience in conjunction with screening for ACEs may help clinicians and patients/families feel more empowered to act. Similarly, more indepth interventions can be integrated into pediatric settings to screen for and address risk and protective factors concurrently. ${ }^{77}$ For example, the Benevolent Childhood Experiences scale screens for positive experiences in a parallel screen designed to accompany the $\mathrm{ACEs}^{78}$ presenting an opportunity to briefly incorporate protective factors into the conversation. By screening for protective factors alongside ACEs, there is potential to identify patterns or create clinically relevant tools to aid providers in providing more precise recommendations tailored to the individual situation. By identifying the positive attributes of various protective factors alongside the potentially stressful ACE exposure, there is an opportunity to focus on the strengths of the child and family and tailor recommendations for next steps. The ability to ask about and then track both risk and resilience together can aid in identifying those children and families that are at particular risk due to limited protective factors.

Finally, screening and surveillance are unlikely to be embraced by clinicians until they also feel empowered to do something helpful for patients with the information they gather. In fact, identifying ACEs without having effective interventions available is unethical at best and potentially harmful. Bringing evidence-based practices-including those noted above such as Triple-P, as well as others such as trauma-focused cognitive-behavioral therapyinto pediatric practice settings is potentially one way to do so, and deserving of further study.

Overall, these emerging trends and ongoing questions argue for caution with any form of individual-level screening. A more conservative approach might instead use region-, institution-, or clinic-wide aggregate screenings, along with screening for protective factors, and efforts to increase the trauma/resiliencecompetencies of health care teams so that they can prevent, identify, assess, and treat ACEs for individual patients holistically within the context of ongoing therapeutic relationshipsagain, this could be accomplished using EHR technologies as described above. Using this approach, aggregate data on the prevalence of ACEs for a given pediatric clinical population could be periodically reported to the health care teams serving that population, along with the prevalence of various protective factors and relevant health outcomes. This information would feed back into the health care teams' collectively readiness to provide enhanced therapeutic relationships, resources, referrals, and interventions for their population as a whole. 


\section{RECOMMENDATIONS}

There is widespread agreement that ACEs are highly prevalent and associated with lifelong health risks that begin during childhood and adolescence, and thus important to identify and ameliorate early. There are also valid questions and obstacles regarding ACE screening and surveillance in pediatrics. To answer these questions and overcome these obstacles, we offer the following recommendations.

For researchers

1. Answer essential questions about whether individual-level universal ACE screening is effective at preventing chronic conditions, or whether it is better used at a population level for surveillance and public health. This can be answered via randomized trials_-ideally multisite implementation/effectiveness trials in real-world settings.

2. Establish ACE screeners' validity, reliability, positive/negative predictive value, and correlation with biomarkers indicative of allostatic load in representative populations, including and/or adapted to cultural and linguistic variables. Such studies should also measure potential harms, downsides, or unintended consequences of ACE screening (e.g., stigmatization, mandated reporting, evoking re-traumatization or other negative feelings in families, and related effects on the clinician-patient relationship ${ }^{50,79,80}$ ).

3. Determine when/how often to rescreen ACEs; who screens for which ACEs and where (e.g., behavioral health, primary care, specialty care); how to best track ACEs over time; and how to best collect contextual details about endorsed items (e.g., timing, duration, imminent risk, caregiver involvement).

4. Determine whether a very limited subset of 1-3 ACEs should be screened for at all pediatric visits (similar to current recommendations regarding domestic violence and depression screening) - that is, by using "first-level" screening for ACEs that occur most frequently and/or co-occur most often with other ACEs, clinicians might readily identify children who should receive comprehensive "second-level" ACE screening and/or referral.

5. Examine interactions between ecological factors and ACEsusing clinical trial methodology when possible (including natural experiments and RCTs) - to identify potential areas for systemic or policy-level intervention (e.g., income inequality ${ }^{81}$ ) that can prevent ACEs and/or improve health outcomes for youth identified with ACEs.

For clinicians

1. Use best practices for psychosocial screening implementation and workflows, for example, using guidelines regarding screening for Social Determinants of Health from the AAP Screening Technical Assistance and Resource Center (STAR). ${ }^{82}$

2. Consider using ACE screening solely as a population- or clinic-wide measure (e.g., to better assess and address community needs) until more research is done to better understand its risks, benefits, and methods. If piloting individual-level ACE screening while awaiting further research, consider using SEEK PQ-R, CYW-ACEQ, or WECARE screeners.

3. Delineate clinical-care processes for children without ACEs (e.g., offering educational resources on prevention and resilience) and those with ACEs (e.g., referral and follow-up). Concise written and oral information should be readily available in multiple languages to provide guidance about risk and protective factors with all families.

4. Determine how ACE information can be input into EHRs (e.g., either through a patient portal or during clinic checkin/rooming). Considerations for these systems should include privacy; how to track ACEs longitudinally; how responses can automatically trigger talking points and/or referral information at the point of care; and how to integrate with EHR shortcuts for easy documentation and referral, including local resources and evidence-based programs.

5. Adopt system-wide, inter-professional trauma-informed approaches to care for children with ACEs: ${ }^{83}$ Realize that ACEs are important for child health; Recognize signs and symptoms of childhood trauma and stress; Respond using resilience-based language and interventions; ${ }^{84}$ and Resist Re-traumatization such as restraints for children undergoing medical procedures.

6. Concurrently identify protective factors and social determinants of health along with ACEs, to better understand strengths and resources. Discuss with all families ways to prevent ACEs, and mitigate/moderate the effects of existing ACEs, using a resilience-informed approach. ${ }^{77}$

7. Consider taking part in care collaboratives that merge screening, early intervention, and prevention in pediatric health care settings, such as Healthy Steps. ${ }^{85}$

8. Clinical training across all pediatric specialties should incorporate trauma- and resilience-informed principles and foster experiential learning in these approaches.

For policymakers

1. Support population-level surveillance for ACEs, for example, by incorporating ACEs into statewide and national surveys of youth and families (e.g., the Youth Risk Behavior Surveillance System).

2. Promote two-generation initiatives and follow-up strategies for ACEs that build human capital (e.g., Futures Without Violence).$^{86,87}$ The efforts can include assessing parents' exposure to childhood adversity and dyadic interventions that address both parent and child health.

3. Support cross-sector workforce development in ACE screening and evidence-based trauma-focused treatments. ${ }^{88,89}$ Successful initiatives have been implemented in many states and communities through home visiting, child welfare, juvenile justice, and educational systems. ${ }^{90-94}$

4. Professional organizations should develop and disseminate practice guidelines for ACE screening and surveillance, in coordination with researchers, clinicians, and educators. These guidelines should take into account, for example, that ACE screening in pediatrics may be viewed favorably by clinicians when framed as universal, confidential, and patient-centered $^{95}$ and that trauma screening protocols add little time to a visit. 63,96

5. Encourage the "unbundling" of service billing to allow reimbursement from insurers for ACE screening occurring in conjunction with a well-child visit. Of note, Early and Periodic Screening, Diagnostic, and Treatment covers a wide range of behavioral health screenings designed for early detection and intervention, and federal guidance to Medicaid state plans suggests inclusion of specific screening for ACEs and exposure to violence. ${ }^{97}$ Additionally, certain states (e.g., Colorado, Vermont, Wisconsin) permit coverage of interventions in pediatric settings such as parental education or assessment, so long as these services cannot be considered treatment for the caregiver. These services, 
however, are often not considered part of routine health care, and thus may not be eligible for financial reimbursement. As such, continued advocacy at the national and state levels is needed to ensure proper payment for the time needed for ACE identification and management.

6. Increase funding and payer coverage for evidence-based treatments. The availability of such resources may be limited in many communities, calling into question whether screening is justified or ethical when proper treatment cannot be assured. Possible state-level remedies, for example, could include expanding state Medicaid benefit package to increase the number of trauma-focused therapies/therapists that are covered.

\section{CONCLUSIONS}

In summary, screening for childhood adversity remains underdeveloped despite evidence that toxic stress during childhood contributes to chronic health conditions. While many pediatric clinicians recognize and understand this, any type of universal, individual-level ACE screening in pediatric health care currently has barriers that need to be addressed. Future ACE screening research must address multiple issues, including screening tools and methods; prospective health outcomes across the lifespan; and individual, family, and system-level factors associated with ACEs, resilience, and response to intervention. Clinical identification of ACEs will have to move away from a screening model that relies solely on a pass/fail or cut-off value; instead, novel solutions such as population-level screening and EHRs can be utilized and hold promise for integrating data about these stressors with factors that might include age, family strengths and resources, cultural values, health, and developmental competence. Pediatricians nonetheless are well positioned to advocate with policymakers about ACEs and resilience as a key step to improving population health. While pediatricians will need alignment with time, evidence, and reimbursement to support ACE identification, they are prevention specialists, and with the help of tools such as EHRs, ready to implement best practices to reduce the societal burden of ACEs.

\section{ACKNOWLEDGEMENTS}

We wish to thank Beth Auslander, Ph.D., M.S., University of Texas Medical Branch Department of Pediatrics, for her contributions to this paper. Funding for this study was covered in part by the Child Health Research Acceleration Through Multisite Planning Pilot funding and the UC Irvine Grant UL1TR001414 and the Children's National Grant UL1TR001876.

\section{AUTHOR CONTRIBUTIONS}

All authors had substantial contributions to the concept, design, and critical review of the literature for this narrative review article; drafting the article and revising it critically for important intellectual content; and have given their final approval of the version to be published. All authors have agreed to its submission; it is not currently being considered for publication by another journal, and it will not be published in the same or similar form in any language without the consent of publishers.

\section{ADDITIONAL INFORMATION}

Competing interests: The authors declare no competing interests.

Publisher's note Springer Nature remains neutral with regard to jurisdictional claims in published maps and institutional affiliations.

\section{REFERENCES}

1. Felitti, V. J. et al. Relationship of childhood abuse and household dysfunction to many of the leading causes of death in adults: the Adverse Childhood Experiences (ACE) Study. Am. J. Prev. Med. 14, 245-258 (1998).

2. Liming, K. W. \& Grube, W. A. Wellbeing outcomes for children exposed to multiple adverse experiences in early childhood: a systematic review. Child Adolesc. Soc. Work J. 35, 317-335 (2018).

3. Felitti, V. J. et al. Relationship of childhood abuse and household dysfunction to many of the leading causes of death in adults. The Adverse Childhood Experiences (ACE) Study. Am. J. Prev. Med. 14, 245-258 (1998).

4. Anda, R. F., Butchart, A., Felitti, V. J. \& Brown, D. W. Building a framework for global surveillance of the public health implications of adverse childhood experiences. Am. J. Prev. Med. 39, 93-98 (2010).

5. Evans, G. W. \& Kim, P. Childhood poverty and health. Psychol. Sci. 18, 953-957 (2007).

6. Chilton, M. \& Rabinowich, J. Toxic stress and child hunger over the life course: three case studies. J. Appl. Res. Child Informing Policy Child Risk 3, 2012 (2012).

7. Richdale, A., Francis, A., Gavidia-Payne, S. \& Cotton, S. Stress, behaviour, and sleep problems in children with an intellectual disability. J. Intellect. Dev. Disabil. 25, 147-161 (2000).

8. Kazak, A. E. et al. An integrative model of pediatric medical traumatic stress. J. Pediatr. Psychol. 31, 343-355 (2006).

9. Thabet, A. A. M., Abed, Y. \& Vostanis, P. Comorbidity of PTSD and depression among refugee children during war conflict. J. Child Psychol. Psychiatry 45, 533-542 (2004)

10. Durkin, M. S., Khan, N., Davidson, L. L., Zaman, S. S. \& Stein, Z. A. The effects of a natural disaster on child behavior: evidence for posttraumatic stress. Am. J. Public Health 83, 1549-1553 (1993).

11. Barnes, A. J., Gilbertson, J. \& Chatterjee, D. Emotional health among youth experiencing family homelessness. Pediatrics 141, e20171767 (2018).

12. Arseneault, L. et al. Bullying victimization uniquely contributes to adjustment problems in young children: a Nationally Representative Cohort Study. Pediatrics 118, 130-138 (2006)

13. Ellis, B. H., MacDonald, H. Z., Lincoln, A. K. \& Cabral, H. J. Mental health of Somali adolescent refugees: the role of trauma, stress, and perceived discrimination. $J$. Consult. Clin. Psychol. 76, 184-193 (2008).

14. Saunderson, E. A. et al. Stress-induced gene expression and behavior are controlled by DNA methylation and methyl donor availability in the dentate gyrus. Proc. Natl Acad. Sci. USA 113, 4830-4835 (2016).

15. McEwen, B. S. Stress, adaptation, and disease. Allostasis and allostatic load. Ann. NY Acad. Sci. 840, 33-44 (1998).

16. Pechtel, P. \& Pizzagalli, D. A. Effects of early life stress on cognitive and affective function: an integrated review of human literature. Psychopharmacology (Berl.) 214, 55-70 (2011).

17. Rutter, M. L. Psychosocial adversity and child psychopathology. Br. J. Psychiatry 174, 480-493 (1999).

18. Ports, K. A. et al. Adverse childhood experiences and suicide risk: toward comprehensive prevention. Am. J. Prev. Med. 53, 400-403 (2017).

19. Sonu, S., Post, S. \& Feinglass, J. Adverse childhood experiences and the onset of chronic disease in young adulthood. Prev. Med. (Balt.) 123, 163-170 (2019).

20. Dimsdale, J. E. Psychological stress and cardiovascular disease. J. Am. Coll. Cardiol. 51, 1237-1246 (2008)

21. Godbout, J. P. \& Glaser, R. Stress-induced immune dysregulation: implications for wound healing, infectious disease and cancer. J. Neuroimmune Pharm. 1, 421-427 (2006).

22. Kerker, B. D. et al. Adverse childhood experiences and mental health, chronic medical conditions, and development in young children. Acad. Pediatr. 15, 510-517 (2015).

23. Garner, A. S. et al. Early childhood adversity, toxic stress, and the role of the pediatrician: translating developmental science into lifelong health. Pediatrics 129, e224-e231 (2012).

24. Bright, M. A., Knapp, C., Hinojosa, M. S., Alford, S. \& Bonner, B. The comorbidity of physical, mental, and developmental conditions associated with childhood adversity: a population based study. Matern. Child Health J. 20, 843-853 (2016).

25. Anda, R. F. et al. The enduring effects of abuse and related adverse experiences in childhood. Eur. Arch. Psychiatry Clin. Neurosci. 256, 174-186 (2006).

26. Burns, B. J. et al. Mental health need and access to mental health services by youths involved with child welfare: a national survey. J. Am. Acad. Child Adolesc. Psychiatry 43, 960-970 (2004).

27. Davis, L., Barnes, A. J., Gross, A. C., Ryder, J. R. \& Shlafer, R. J. Adverse childhood experiences and weight status among adolescents. J. Pediatr. 204, 71-76.e1 (2019).

28. Conn, A.-M. et al. Parental perspectives of screening for adverse childhood experiences in pediatric primary care. Fam. Syst. Health 36, 62-72 (2018).

29. American Academy of Pediatrics Task Force on Mental Health. Appendix S4: the case for routine mental health screening. Pediatrics 125, S133-S139 (2010). 
30. Szilagyi, M. et al. Factors associated with whether pediatricians inquire about parents' adverse childhood experiences. Acad. Pediatr. 16, 668-675 (2016).

31. Kerker, B. D. et al. Detection and treatment of mental health issues by pediatric PCPs in New York state: an evaluation of Project TEACH. Psychiatr. Serv. 66, 430-433 (2015).

32. Brown, J. D., King, M. A. \& Wissow, L. S. The central role of relationships with trauma-informed integrated care for children and youth. Acad. Pediatr. 17, S94-S101 (2017)

33. Blair, C. \& Raver, C. C. Poverty, stress, and brain development: new directions for prevention and intervention. Acad. Pediatr. 16, S30-S36 (2016).

34. Chiang, J. J., Chen, E. \& Miller, G. E. Midlife self-reported social support as a buffer against premature mortality risks associated with childhood abuse. Nat. Hum. Behav. 2, 261 (2018).

35. Masten, A. S. Ordinary magic: resilience processes in development. Am. Psychol. 56, 227-238 (2001)

36. Slopen, N., McLaughlin, K. A. \& Shonkoff, J. P. Interventions to improve cortisol regulation in children: a systematic review. Pediatrics 133, 312-326 (2014).

37. Fisher, P. A., Van Ryzin, M. J. \& Gunnar, M. R. Mitigating HPA axis dysregulation associated with placement changes in foster care. Psychoneuroendocrinology 36 531-539 (2011).

38. Burke, N. J., Hellman, J. L., Scott, B. G., Weems, C. F. \& Carrion, V. G. The impact of adverse childhood experiences on an urban pediatric population. Child Abuse Negl. 35, 408-413 (2011).

39. Bethell, C., Davis, M., Gombojav, N., Stumbo, S. \& Powers, K. Issue brief: a national and across state profile on adverse childhood experiences among children and possibilities to heal and thrive. Johns Hopkins Bloomberg School of Public Health, October 2017. http://www.cahmi.org/projects/adverse-childhood-experiencesaces/. Accessed 10/17/19.

40. Kerker, B. D. et al. Identifying maternal depression in pediatric primary care. J. Dev. Behav. Pediatr. 37, 113-120 (2016).

41. Horwitz, S. M. et al. Barriers to the identification and management of psychosocial issues in children and maternal depression. Pediatrics 119, e208-e218 (2007).

42. Williams, J., Klinepeter, K., Palmes, G., Pulley, A. \& Foy, J. M. Diagnosis and treatment of behavioral health disorders in pediatric practice. Pediatrics 114, 601-606 (2004)

43. Horwitz, S. M. et al. Barriers to the identification and management of psychosocial problems: changes from 2004 to 2013. Acad. Pediatr. 15, 613-620 (2015).

44. Bright, M. A., Thompson, L., Esernio-Jenssen, D., Alford, S. \& Shenkman, E. Primary care pediatricians' perceived prevalence and surveillance of adverse childhood experiences in low-income children. J. Health Care Poor Underserved 26, 686-700 (2015).

45. Dube, S. R. Continuing conversations about adverse childhood experiences (ACEs) screening: a public health perspective. Child Abuse Negl. 85, 180-184 (2018).

46. Wilson, J. M. G. \& Jungner, G. Principles and Practice of Screening for Disease (26-39) (World Health Organization, Geneva, 1968).

47. Finkelhor, D., Shattuck, A., Turner, H. \& Hamby, S. Improving the Adverse Childhood Experiences Study Scale. JAMA Pediatr. 167, 70-75 (2013).

48. Niel Van, C. et al. Adverse events in children: predictors of adult physical and mental conditions. J. Dev. Behav. Pediatr. 35, 549-551 (2014).

49. Finkelhor, D., Shattuck, A., Turner, H. \& Hamby, S. A revised inventory of adverse childhood experiences. Child Abus. Negl. 48, 13-21 (2015).

50. Finkelhor, D. Screening for adverse childhood experiences (ACEs): cautions and suggestions. Child Abuse Negl. 85, 174-179 (2018).

51. Flaherty, E. G. \& Stirling, J. American Academy of Pediatrics. Committee on Child Abuse and Neglect. Clinical report-the pediatrician's role in child maltreatment prevention. Pediatrics 126, 833-841 (2010)

52. Garner, A. S. et al. Early childhood adversity, toxic stress, and the role of the pediatrician: translating developmental science into lifelong health. Pediatrics 129, e224-e231 (2012)

53. Bernstein, D. P. et al. Development and validation of a brief screening version of the Childhood Trauma Questionnaire. Child Abuse Negl. 27, 169-190 (2003).

54. Grasso, D. J., Felton, J. W. \& Reid-Quiñones, K. The Structured Trauma-Related Experiences and Symptoms Screener (STRESS). Child Maltreat. 20, 214-220 (2015).

55. Brier, J. Trauma Symptom Checklist for Children|The National Child Traumatic Stress Network [cited 13 Apr 2019]. https://www.nctsn.org/measures/traumasymptom-checklist-children (1996).

56. Hamby, S. L., Finkelhor, D., Ormrod, R., Turner, H. \& Hamby, L. The Juvenile Victimization Questionnaire (JVQ): administration and scoring manual [cited 13 Apr 2019]. http://www.unh.edu/ccrc/pdf/CV55 2004.pdf (2004).

57. Choi, K. R. et al. Validation of the traumatic events screening inventory for ACEs. Pediatrics 143, e20182546 (2019).
58. Perrin, E. C., Sheldrick, C., Visco, Z. \& Mattern, K. The Survey of Well-being of Young Children (SWYC) User's Manual. version 1 (Floating Hospital for Children at Tufts Medical Center, Boston, 2016).

59. Burke Harris, N. \& Renschler, T. Center for Youth Wellness ACE-Questionnaire (CYW ACE-Q Child, Teen, Teen SR). version 7 (Center for Youth Wellness, San Francisco, 2015).

60. Oh, D. L. et al. Review of tools for measuring exposure to adversity in children and adolescents. J. Pediatr. Health Care 32, 564-583 (2018).

61. Mersky, J. P., Janczewski, C. E. \& Topitzes, J. Rethinking the measurement of adversity: moving toward second-generation research on adverse childhood experiences. Child Maltreat. 22, 58-68 (2017).

62. Dubowitz, H., Lane, W. G., Semiatin, J. N. \& Magder, L. S. The SEEK model of pediatric primary care: can child maltreatment be prevented in a low-risk population? Acad. Pediatr. 12, 259-268 (2012).

63. Garg, A. et al. Improving the management of family psychosocial problems at low-income children's well-child care visits: the WE CARE Project. Pediatrics 120, 547-558 (2007).

64. Garg, A., Toy, S., Tripodis, Y., Silverstein, M. \& Freeman, E. Addressing social determinants of health at well child care visits: a cluster RCT. Pediatrics 135, e296-e304 (2015).

65. Tonmyr, L., Draca, J., Crain, J. \& MacMillan, H. L. Measurement of emotional/ psychological child maltreatment: a review. Child Abuse Negl. 35, 767-782 (2011).

66. Milne, L. \& Collin-Vézina, D. Assessment of children and youth in child protective services out-of-home care: an overview of trauma measures. Psychol. Violence 5, 122-132 (2015).

67. SEEK (Safe Environment for Every Kid). SEEK Parent Questionnaire-R (PQ-R) formerly the PQ or PSQ [cited 21 Apr 2019]. https://www.seekwellbeing.org/theseek-parent-questionnaire (2018).

68. Lingras, K., Greifer, M., Sheikh, K. \& Fabre, B. Adverse childhood experiences (ACEs) and trauma in young children: what we know and what we can do. Child Ment. Health eReview 1-22 (2019).

69. American Academy of Pediatrics EQIPP [cited 21 Apr 2019]. https://eqipp.aap.org/ (2019).

70. American Board of Pediatrics Practice Improvement Modules [cited 21 Apr 2019]. https://www.abp.org/content/online-performance-improvement-modules-pims (2019).

71. The Pediatric Integrated Care Collaborative [cited 21 Apr 2019]. https://picc.jhu. edu/ (2019).

72. Oral, R. et al. Adverse childhood experiences and trauma informed care: the future of health care. Pediatr. Res. 79, 227-233 (2016).

73. Karatekin, C., Almy, B., Mason, S. M., Borowsky, I. \& Barnes, A. Documentation of child maltreatment in electronic health records. Clin. Pediatr. (Philos.) 57 1041-1052 (2018).

74. Sun, J. et al. Mothers' adverse childhood experiences and their young children's development. Am. J. Prev. Med. 53, 882-891 (2017).

75. Purewal Boparai, S. K. et al. Ameliorating the biological impacts of childhood adversity: a review of intervention programs. Child Abuse Negl. 81, 82-105 (2018).

76. Shakiba, N., Ellis, B. J., Bush, N. R. \& Boyce, W. T. Biological sensitivity to context: a test of the hypothesized U-shaped relation between early adversity and stress responsivity. Dev. Psychopathol. 1-20 (2019). https://doi.org/10.1017/ S0954579419000518 [Epub ahead of print].

77. Masten, A. \& Barnes, A. Resilience in children: developmental perspectives. Children 5, 98-114 (2018).

78. Narayan, A. J., Rivera, L. M., Bernstein, R. E., Harris, W. W. \& Lieberman, A. F. Positive childhood experiences predict less psychopathology and stress in pregnant women with childhood adversity: a pilot study of the benevolent childhood experiences (BCEs) scale. Child Abuse Negl. 78, 19-30 (2018).

79. Van Haeringen, A. R., Dadds, M. \& Armstrong, K. L. The child abuse lottery-will the doctor suspect and report? Physician attitudes towards and reporting of suspected child abuse and neglect. Child Abuse Negl. 22, 159-169 (1998).

80. Vulliamy, A. P. \& Sullivan, R. Reporting child abuse: pediatricians' experiences with the child protection system. Child Abuse Negl. 24, 1461-1470 (2000).

81. Halfon, N., Larson, K., Son, J., Lu, M. \& Bethell, C. Income inequality and the differential effect of adverse childhood experiences in US children. Acad. Pediatr. 17, S70-S78 (2017)

82. American Academy of Pediatrics Screening Technical Assistance and Resource Center. [cited 21 Apr 2019]. https://www.aap.org/en-us/advocacy-and-policy/aaphealth-initiatives/Screening/Pages/default.aspx (2019).

83. Karatekin, C. \& Almy, B. Adverse childhood experiences 101: a primer for clinicians. Creat. Nurs. 25, 87-102 (2019).

84. Traub, F. \& Boynton-Jarrett, R. Modifiable resilience factors to childhood adversity for clinical pediatric practice. Pediatrics 78, 19-30 (2017).

85. Kaplan-Sanoff, M. \& Briggs, R. D. in Integrated Early Childhood Behavioral Health in Primary Care (ed. Briggs, R. D.) 71-83 (Springer, 2016). 
86. Flynn, A. B. et al. Primary care interventions to prevent or treat traumatic stress in childhood: a systematic review. Acad. Pediatr. 15, 480-492 (2015).

87. Heckman, J. J., James, J., Krueger, A. B. \& Friedman. B. M. Inequality in America: What Role for Human Capital Policies? (MIT Press, 2003)

88. Lang, J. M. et al. Trauma screening in child welfare: lessons learned from five states. J. Child Adolesc. Trauma 10, 405-416 (2017).

89. Lang, J. M., Campbell, K., Shanley, P., Crusto, C. A. \& Connell, C. M. Building capacity for trauma-informed care in the child welfare system: initial results of a statewide implementation. Child Maltreat. 21, 113-124 (2016).

90. Adirim, T. \& Supplee, L. Overview of the Federal Home Visiting Program. Pediatrics 132, S59-S64 (2013).

91. Mckelvey, L. et al. Examing long-term effects of an infant mental health homebased Early Head Start program on family strengths and resilience. Infant Ment. Health J. 36, 353-365 (2015).

92. Fox, B. H., Perez, N., Cass, E., Baglivio, M. T. \& Epps, N. Trauma changes everything: examining the relationship between adverse childhood experiences and serious, violent and chronic juvenile offenders. Child Abuse Negl. 46, 163-173 (2015).
93. Eklund, K. \& Rossi, E. Guidance for trauma screening in schools a product of the defending childhood state policy initiative. (2016). https://www.nasponline.org/ x37269.xml. Accessed 10/17/19.

94. Gonzalez, A., Monzon, N., Solis, D., Jaycox, L. \& Langley, A. K. Trauma exposure in elementary school children: description of screening procedures, level of exposure, and posttraumatic stress symptoms. School Ment. Health 8, 77 (2016).

95. Wissow, L. S. et al. Universal mental health screening in pediatric primary care: a systematic review. J. Am. Acad. Child Adolesc. Psychiatry 52, 1134-1147.e23 (2013).

96. Dubowitz, H., Feigelman, S., Lane, W. \& Kim, J. Pediatric primary care to help prevent child maltreatment: the Safe Environment for Every Kid (SEEK) Model. Pediatrics 123, 858-864 (2009).

97. US Department of Health and Human Services: Guidance to State Directors of Medicaid [cited 21 Apr 2019]. http://www.medicaid.gov/Federal-Policy-Guidance/ Downloads/SMD-13-07-11.pdf. (2013).

98. Pediatric Integrated Care Collaborative (PICC). PICC Toolkit 1.0: Improving the capacity of primary care to serve children and families experiencing trauma and chronic stress. https://picc.jhu.edu/the-toolkit.html. Johns Hopkins University Bloomberg School of Public Health (2016). Accessed 10/17/19. 